\title{
Fabrication of sub-100 nm IDT SAW devices on insulating, semiconducting and conductive substrates
}

\author{
G.F. Iriarte*, J.G. Rodriguez-Madrid, F. Calle \\ Instituto de Sistemas Optoelectrónicos y Microtecnología, Universidad Politécnica de Madrid, ETSI Telecomunicaciôn, Ciudad Universitaria s/n, 28040 Madrid, Spain
}

\begin{abstract}
A B S T R A C T
This work describes the electron-beam (e-beam) lithography process developed to manufacture nano interdigital transducers (IDTs) to be used in high frequency (GHz) surface acoustic wave (SAW) applications. The combination of electron-beam (e-beam) lithography and lift-off process is shown to be effective in fabricating well-defined IDT finger patterns with a line width below $100 \mathrm{~nm}$ with a good yield. Working with insulating piezoelectric substrates brings about e-beam deflection. It is also shown how a very thin organic anti-static layer works well in avoiding this charge accumulation during e-beam lithography on the resist layer. However, the use of this anti-static layer is not required with the insulating piezoelectric layer laying on a semiconducting substrate such as highly doped silicon. The effect of the e-beam dose on a number of different layers (of insulating, insulating on semiconducting, semiconducting, and conductive natures) is provided. Among other advantages, the use of reduced e-beam doses increases the manufacturing time.

The principal aim of this work is to explain the interrelation among e-beam dose, substrate nature and IDT structure. An extensive study of the e-beam lithography of long IDT-fingers is provided, in a wide variety of electrode widths, electrode numbers and electrode pitches. It is worthy to highlight that this work shows the influence of the e-beam dose on five substrates of different conductive nature.
\end{abstract}

\section{Introduction}

Surface acoustic wave (SAW) devices are now essential components in mobile communication and radar systems, among other applications. To achieve higher frequency demands the development of reliable lithography techniques, able to produce interdigital transducers (IDT) of several hundred periods with a reduced wavelength.

Smith (1974) explains thoroughly how to develop SAW devices by different lithography techniques as photolithography, electron lithography and X-ray lithography, comparing advantages and disadvantages of each technique. At that time, photolithography was the most convenient and economical technique, but scanningelectron-beam lithography was already suggested to be the future solution for complex high-resolution patterns. Yamanouchi et al. (1988) produced $10 \mathrm{GHz}$ SAW devices based on $\mathrm{LiNbO}_{3}$ with $85 \mathrm{~nm}$ wide e-beam lithography IDT-fingers. Contrary to thin films, this substrate cannot be integrated in the CMOS technology.

The piezoelectric properties of the III-nitride semiconductors have attracted much attention in the last decade for the fabrication of high frequency acoustic wave devices, by means of electron beam lithography and lift-off process. Due to the poor electrical conductivity and low chemical reactivity of these materials (in particular AIN), Palacios et al. (2002) tested several resist/metal multilayers, both by experiments and simulations, the most successful being the resist/metal/semiconductor and the resist/metal/resist/semiconductor schemes. Hatekeyama et al. (2004) achieved IDT-fingers of $180 \mathrm{~nm}$ wide; reduced size designs of $100 \mathrm{~nm}$ wide showed a poor production yield. Recently, SAW devices at $5 \mathrm{GHz}$ have been fabricated on AlN/Si and $\mathrm{GaN} / \mathrm{Si}$ structures by Neculoiu et al. (2009) and Müller et al. (2010), respectively. These high frequency devices in general show high losses likely due to the design of the test structures or impedance mismatch.

In addition to the lithography, the use of materials (layers and/or substrates) with high stiffness coefficients and low density also increases the device frequency. The most typical example is the AlN/diamond combination, used by Iriarte (2009) to fabricate SAW devices at $1 \mathrm{GHz}$ with photolithographic IDT. E-beam devices operating at $5 \mathrm{GHz}$ were developed by Kirsh et al. (2006). Assouar et al. (2007) also used the AlN/diamond structure to achieve SAW devices at $8 \mathrm{GHz}$ with good temperature stability.

In summary, compared to the conventional optical lithography, e-beam lithography is most useful and practical for laboratory work, offering a better line resolution and a cost effective solution for iteration design-test structure. However, e-beam lithography 


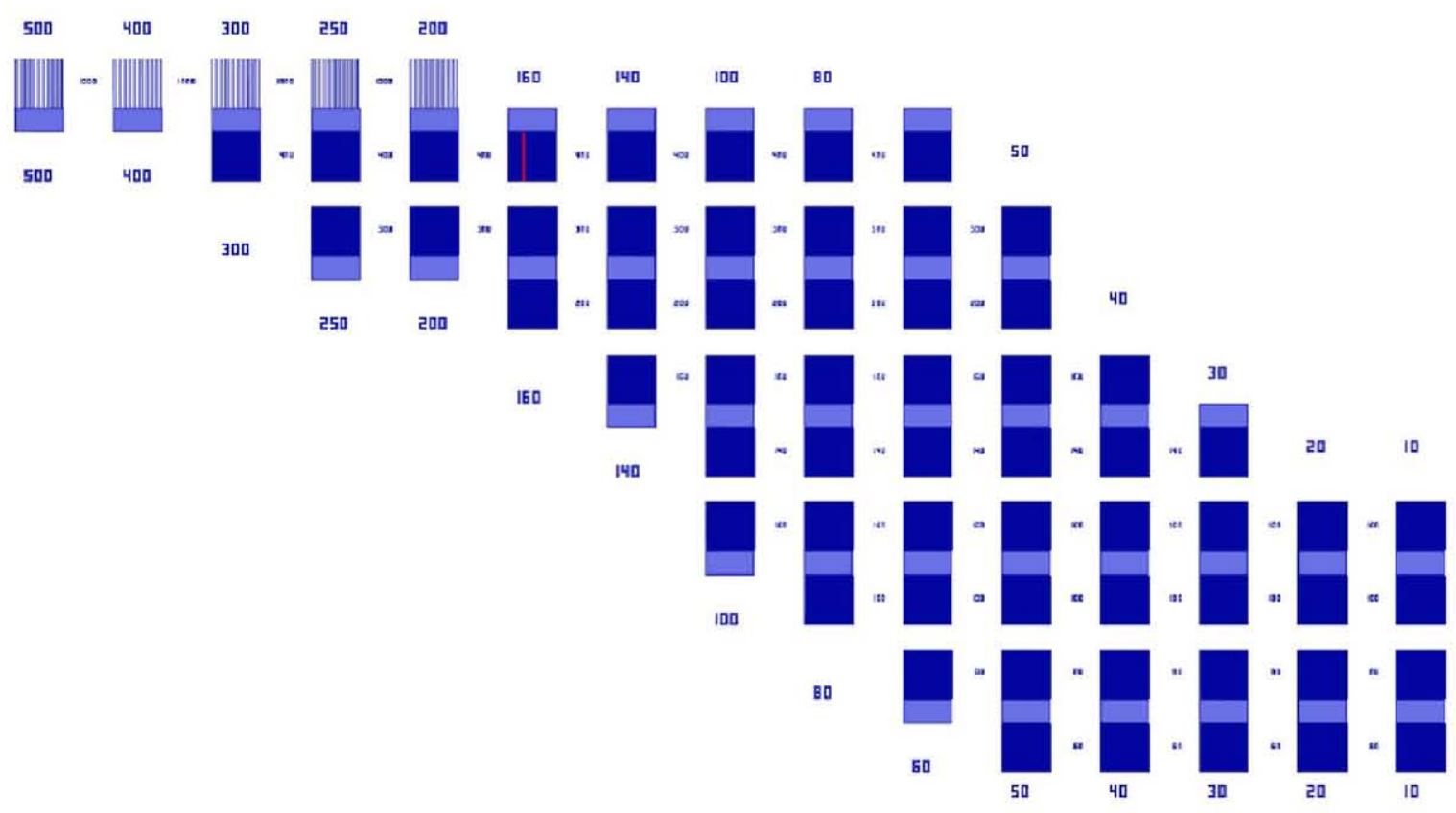

Fig. 1. Matrix of IDTs used in this work.

poses some disadvantages from the technical side, and charge accumulation and dose effects must be carefully considered. In this work, we have applied e-beam lithography and lift-off techniques for the fabrication of IDTs on five substrate types of different conductivity. Through this process, it is shown that well-defined IDT finger patterns with line widths below $100 \mathrm{~nm}$ can be fabricated at a good yield in conductive or semiconducting layers, but also on thin insulating layers deposited on conductive or semiconducting substrates. It is also shown that the use of a very thin organic antistatic layer is effective in minimizing the charge up issue for e-beam exposure on insulating substrates. As a result of this procedure, a SAW device with narrow electrode lines has been fabricated on AlN/diamond which operates at frequencies higher than $10 \mathrm{GHz}$.

\section{Experimental procedure}

The effect of the e-beam dose has been studied on five substrates of different character as regard the exposing e-beam. The substrates studied are the following ones:

\section{Platinum (Pt): conducting layer}

Silicon (Si): semiconducting layer

AIN on Si: piezoelectric on semiconducting layer

AlN on $\mathrm{Al}_{2} \mathrm{O}_{3}$ : piezoelectric on insulating layer

$\mathrm{Al}_{2} \mathrm{O}_{3}$ (alumina): insulating layer

The e-beam dose time has been varied in the range of $0.40-0.70 \mu \mathrm{s}$ in $0.05 \mu \mathrm{s}$ steps. The field size used is of $600 \mu \mathrm{m} \times 600 \mu \mathrm{m}$; the amount of dots per field is 60.000. Dose time (Dt) in this work refers to the time the e-beam spends on each of these dots.

Table 1

E-beam lithography conditions.

\begin{tabular}{ll}
\hline Resist & ZEP520-12 \\
Resist thickness & $150 \mathrm{~nm}$ \\
Acc. voltage & $50 \mathrm{kV}$ \\
Beam current & $500 \mathrm{pA}$ \\
Resist sens. & $220 \mu \mathrm{C} / \mathrm{cm}^{2}$ \\
Field size & $600 \mu \mathrm{m} \times 600 \mu \mathrm{m}$ \\
\hline
\end{tabular}

In order to properly study the achievable resolution on each substrate, a matrix of IDTs has been used. Within this matrix, the line width goes from $300 \mathrm{~nm}$ down to $10 \mathrm{~nm}$ in steps of $20 \mathrm{~nm}$, whereas the finger pitch is varied from $500 \mathrm{~nm}$ down to $10 \mathrm{~nm}$ in $20 \mathrm{~nm}$ steps. The length of the electrodes is $20 \mu \mathrm{m}$. Fig. 1 shows its whole appearance.

The resist ZEP520 (diluted 1:2) was spinned at $5000 \mathrm{rpm}$ during $1 \mathrm{~min}$, which resulted in a resist thickness of approximately $150 \mathrm{~nm}$. After the e-beam lithography step, a thin $\mathrm{Cr} / \mathrm{Al}$ layer $(5 \mathrm{~nm} / 20 \mathrm{~nm}$ ) was evaporated on each sample. Chrome $(\mathrm{Cr})$ was used to enhance the adhesion of the Al layer to the underlying substrate. After evaporation, the samples were introduced on a 1-methyl-2-pyrrolidone bath at $85^{\circ} \mathrm{C}$ for $2 \mathrm{~h}$ to lift-off the resist and the metal on top of it. The lines remaining on the substrate were analyzed on the SEM, both after resist development as well as after the lift-off.

Both the exposure and imaging analysis after the resist development were performed by means of an e-beam lithography system based on a field-emission type filament (CRESTEC CABL-9500C). The system has a $50 \mathrm{keV}$ column and resolves a minimum line width of $10 \mathrm{~nm}$ with a minimum beam diameter of $2 \mathrm{~nm}$. The lowest AC

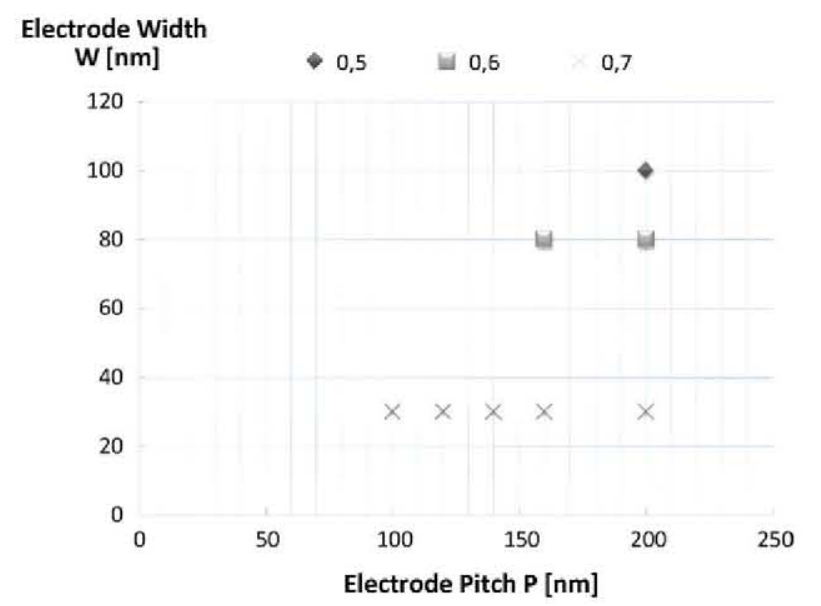

Fig. 2. Minimum dose times ( $\mu s$ ) suggested for e-beam lithography of different IDTs on Pt substrates. 

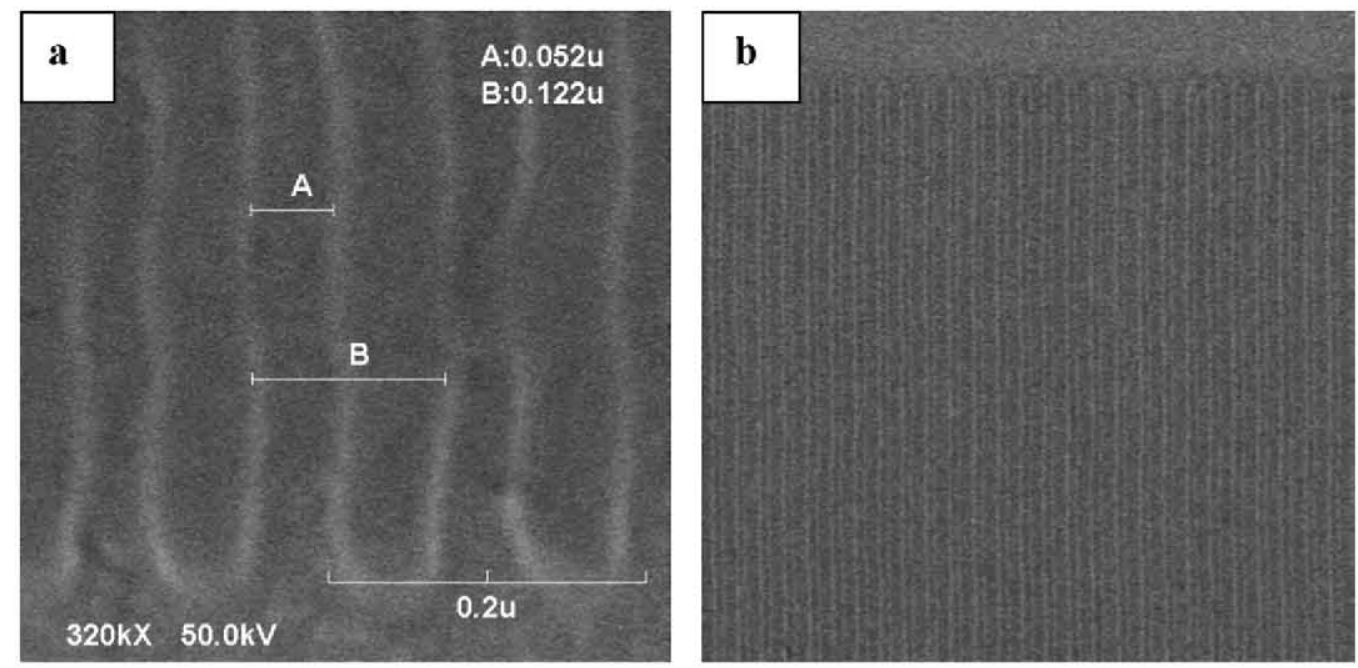

Fig. 3. (a) $50 \mathrm{~nm}$ and (b) $30 \mathrm{~nm}$ wide electrodes with (on the pattern) $100 \mathrm{~nm}$ pitch on a platinum (Pt) substrate, patterned with $65 \mu \mathrm{s}$ and $70 \mu \mathrm{s}$ doses times, respectively.

voltage is $5 \mathrm{kV}$, and the beam current can be varied from $5 \mathrm{pA}$ to $100 \mathrm{nA}$. A beam position stability of $\pm 30 \mathrm{~nm} / 5 \mathrm{~h}$ is guaranteed as well as a $2 \mathrm{~nm}$ work stage resolution.

When working with insulating substrates, an organic anti-static layer (Espacer 300Z, Showa Denko) is used to avoid the charge accumulation. The layer scarcely influences the resolution of the e-beam lithography when this is done on semiconducting or metallic substrates, but it strongly influences the minimum resolution when working directly on insulating substrates.

Details of the process are as follow:

1. Spin coating of e-beam resist (ZEP520, Zeon) on a cleaned substrate: The thickness of the resist layer is chosen properly considering the trade-off between the required resolution and the electrode thickness. That is, it is empirically known that the resist layer thickness should be at least $100 \mathrm{~nm}$ thicker than the metal thickness for the following lift-off process, though the resolution increases with a decrease in the resist layer thickness.

2. Hard-baking: The resist was hard baked for 2 min at $190^{\circ} \mathrm{C}$.

3. Spin coating of antistatic layer (Espacer 300Z, Showa Denko), when required (only for insulating substrates): The thickness of the anti-static layer is of about $20 \mathrm{~nm}$ when spinned at $3000 \mathrm{rpm}$ for $60 \mathrm{~s}$.

4. E-beam exposure: The dose amount should be decided properly by taking into account the substrate material. See Table 1 for other parameters used.

5. When required, removal of anti-static layer: This process is performed by simply immersing the specimen in distilled water for a few seconds.

6. Resist development by dipping in ZED-N50 (Zeon) developer for $60 \mathrm{~s}$ and rinse by blowing with $\mathrm{N}_{2}$ for a few seconds.

7. Deposition of a very thin $(5 \mathrm{~nm}) \mathrm{C}_{\mathrm{r}}$ film for adhesion improvement and a $20 \mathrm{~nm}$ thin $\mathrm{Al}$ film on the specimen by vacuum evaporation.

8. Removal of the resist layer (lift-off) by immersion in 1-methyl2-pyrrolidinone at $85^{\circ} \mathrm{C}$.

\section{Results and discussion}

The results obtained on each of the five different substrates are analyzed either after resist development or after lift-off for reasons explained in each particular case. In the following, the IDT will be named after the line width or pitch used; e.g., W100 stands for electrode line width of $100 \mathrm{~nm}$ and $P 200$ stands for electrode line pitch of $200 \mathrm{~nm}$, where pitch is defined here as the distance resulting from the sum of the electrode width and the space in-between the sides of two adjacent electrodes facing each other.

\subsection{Platinum (Pt)}

Fig. 2 shows the range of times in $\mu$ s suggested for several combinations of IDT line width $(W)$ and pitch $(P)$. Some results are shown in Fig. 3.

E-beam times of $0.40 \mu \mathrm{s}$ and $0.45 \mu \mathrm{s}$ were too low and did not delivered good results. A $0.50 \mu$ s dose time was enough for $W 100: P 200$, but $W 100: P 160$ was not resolved even using the longest time $(0.70 \mu \mathrm{s})$. The $W 80: P 160$ ratio required a $D$ t of at least $0.60 \mu \mathrm{s}$.

In summary, for lithography on Pt substrate, the best results are usually achieved using dose times of $0.70 \mu \mathrm{s}$. Shorter times as low as $0.50 \mu$ s were good enough for $100 \mathrm{~nm}$ wide electrodes on a $200 \mathrm{~nm}$ pitch, and could be used to speed up the e-beam lithography process. Line widths of $20 \mathrm{~nm}$ and below would require further optimization.

\subsection{Silicon (Si)}

As shown in Fig. 4, Dt of at least $0.65 \mu$ s is suggested for W100:P200. Line widths down to $20 \mathrm{~nm}$ could be obtained on a $100 \mathrm{~nm}$ pitch, but the electrode edges were not resolved. Lines

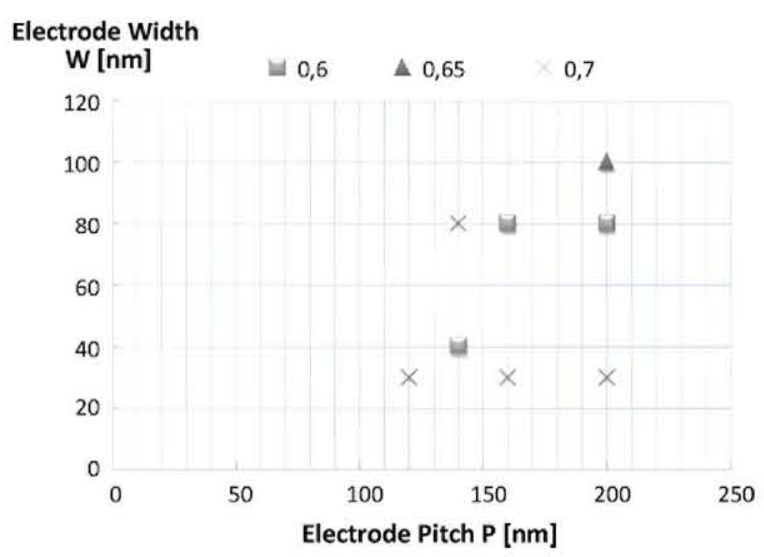

Fig. 4. Minimum Dt ( $\mu s)$ for e-beam lithography suggested for several IDTs on Si substrates. 

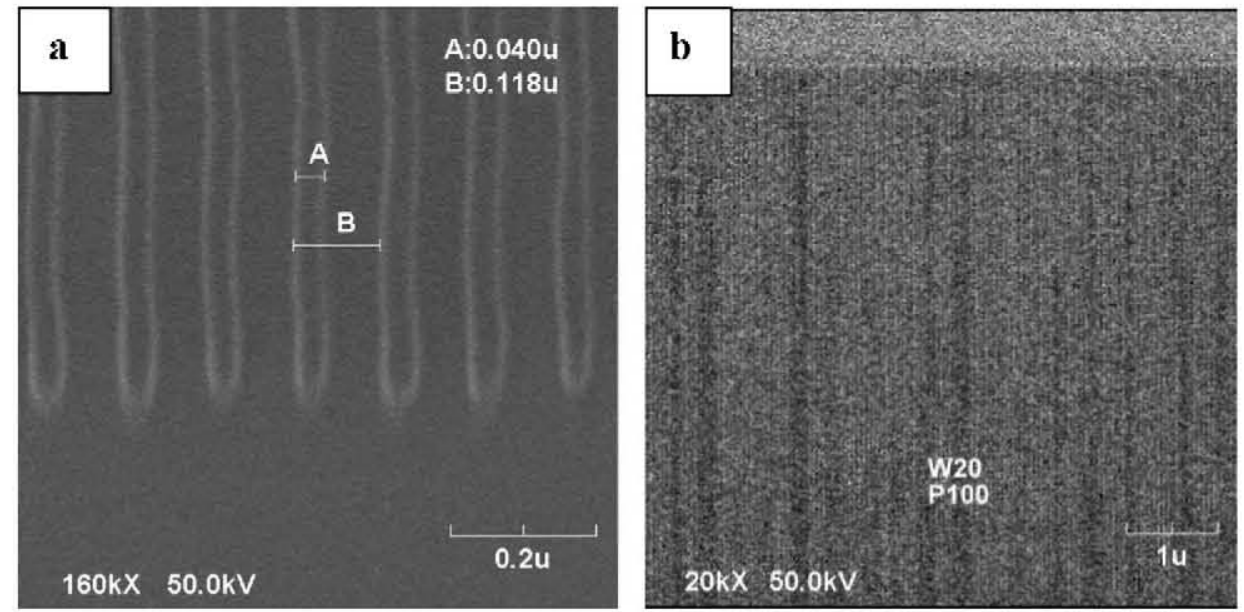

Fig. 5. Silicon substrate: (a) $40 \mathrm{~nm}$ electrodes on a (on the pattern) $100 \mathrm{~nm}$ pitch ( $60 \mu \mathrm{s}$ ). (b) $20 \mathrm{~nm}$ electrodes on a $100 \mathrm{~nm}$ pitch showing some discontinuity ( $70 \mu \mathrm{s}$ ).

below $20 \mathrm{~nm}$ in width require special optimization. Some results are shown in Fig. 5.

\subsection{AlN on $\mathrm{Si}$}

In this section we describe the patterning of IDTs on AIN layers, $1.5 \mu \mathrm{m}$ thick, deposited by reactive sputtering on Si substrates. Despite the non-conductive nature of the AIN film, the AIN/Si system does not require the use of an antistatic layer for successful e-beam lithography.

However, due to charging effects, SEM cannot be used to analyze the sample after resist development. We tried to spin the antistatic layer to a higher speed in order to make it thinner (less than $20 \mathrm{~nm}$ ) in an attempt to analyze the lines obtained. The charging effect could not be avoided; moreover, the resist tends to get slightly wider after a long exposure to the e-beam during SEM analysis. Therefore, no SEM analysis of the "as developed" samples could be done. Instead, the lift-off step was done, and the analysis of the results by SEM was used to determine the achievable electrode width-electrode pitch ratio under each dose time. As shown in Fig. 6, higher times are required on the AlN/Si system as compared to the Pt or Si systems.

Dose times below $0.65 \mu$ s are not recommended on the AlN/Si substrate system, at least without using an antistatic layer.

Line widths below $50 \mathrm{~nm}$ require special optimization and/or the use of an antistatic layer. The manufacture of $100 \mathrm{~nm}$ wide

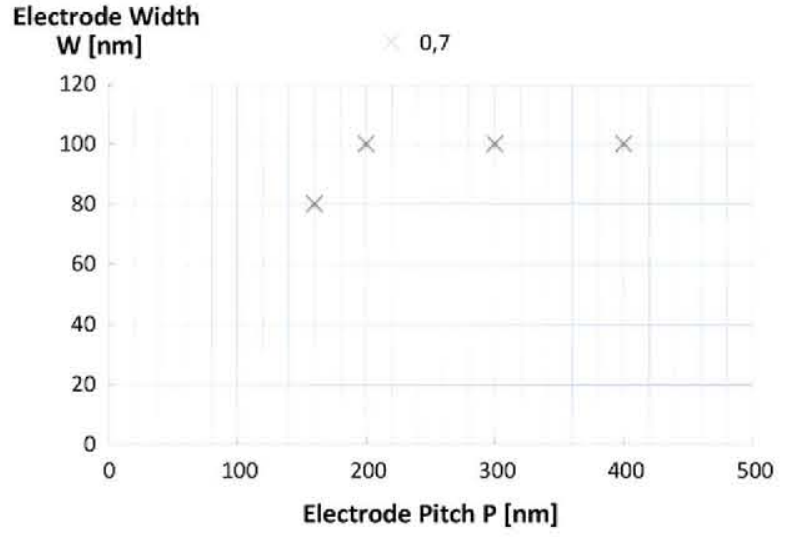

Fig. 6. Minimum Dt ( $\mu s)$ suggested for e-beam patterning of IDTs on AIN/Si struc tures.

electrodes required pitches above $200 \mathrm{~nm}$, but $80 \mathrm{~nm}$ wide lines at $160 \mathrm{~nm}$ pitch could be defined by lift-off at very good yield (Fig. 7).

\section{4. $\mathrm{Al}_{2} \mathrm{O}_{3}$ and $\mathrm{AlN} / \mathrm{Al}_{2} \mathrm{O}_{3}$}

When fabricating IDTs on insulating materials by e-beam lithography, an antistatic polymer layer such as the Espacer 300Z (Showa Denko) should be used. This is the case discussed in this section
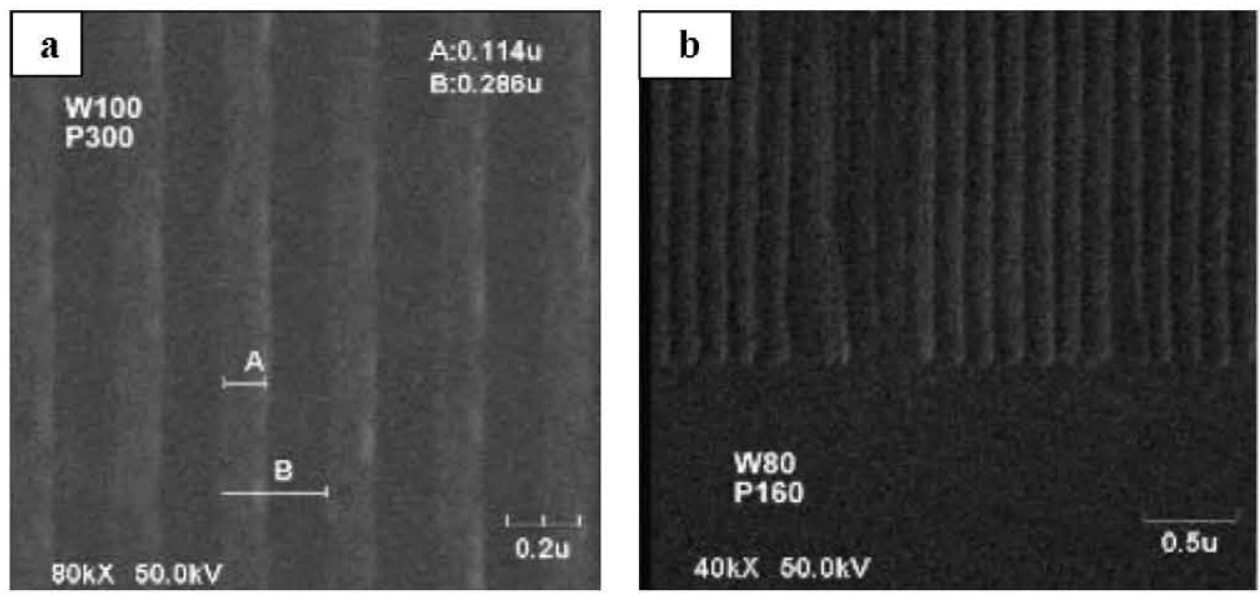

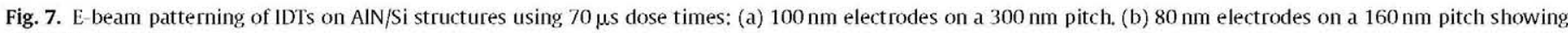
some discontinuity. 

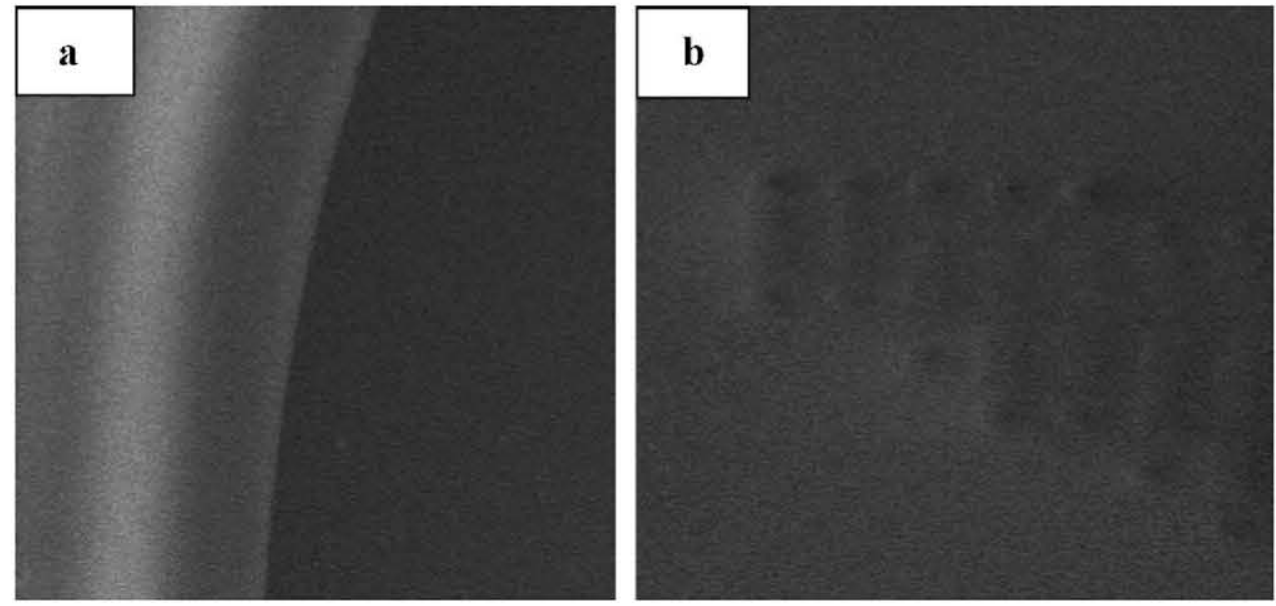

Fig. 8. (a) Charging effect shown on image's left half. (b) Use of Espacer 300Z (Showa Denko) for SEM analysis after resist development.

for the lithography and lift-off on $\mathrm{Al}_{2} \mathrm{O}_{3}$ substrate and $\mathrm{AlN} / \mathrm{Al}_{2} \mathrm{O}_{3}$ structures. Actually, insulating substrates show a difficulty for SEM analysis after resist development of the achieved resolution due to charging effects. Fig. 8 a shows a difference between an area where the antistatic layer is present (right half of the image), and an area where it was not used (left half of the image). Due to charging effects, SEM cannot be used directly to analyze the sample after resist development. Although the Espacer $300 \mathrm{Z}$ layer is only $20 \mathrm{~nm}$ thick, neither can it be used for SEM analysis after resist development. The $20 \mathrm{~nm}$ thin layer does not follow the contour of the developed resist lines, as shown in Fig. 8b. We tried to spin the antistatic layer to a higher speed in order to make it thinner $(<20 \mathrm{~nm})$ in an attempt to analyze the lines developed. The charging effect could not be avoided and hence no SEM analysis of the "as developed" samples could be done. Moreover, the resist tends to get slightly wider after a prolonged exposure to the e-beam during SEM analysis. For these reasons, the attempt to analyze the "as developed" structures was abandoned.

Instead, the lift-off step was done and the results of it, which could indeed be inspected under the SEM, were used to determine the achievable electrode width-electrode pitch ratio under each dose time.

As shown in Fig. 9, this substrate system showed to be more critical regarding the pitch. Thus, lift-off of electrode pitches below $100 \mathrm{~nm}$ could not be successfully done for sub-100 nm width

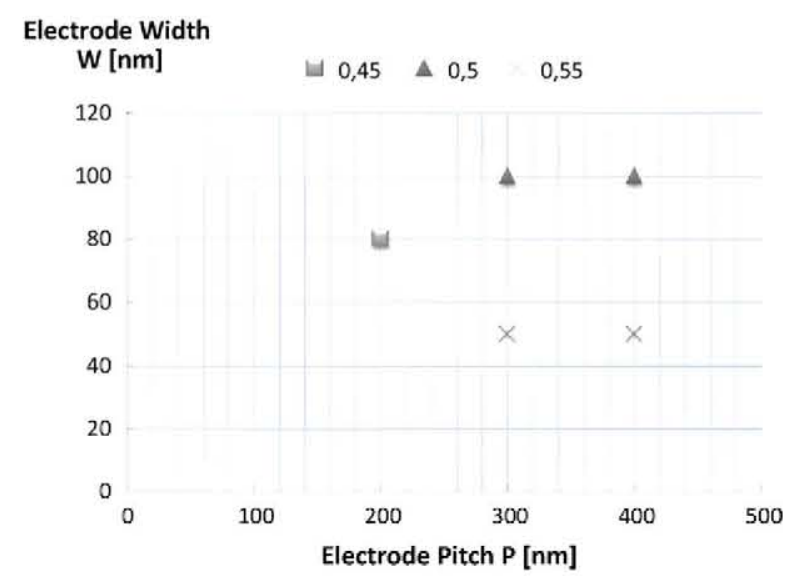

Fig. 9. Minimum doses ( $\mu$ s) suggested on $\mathrm{AIN} / \mathrm{Al}_{2} \mathrm{O}_{3}$ and $\mathrm{Al}_{2} \mathrm{O}_{3}$ substrate system.

electrodes. However, it is remarkable to see how widths as small as $60 \mathrm{~nm}$ and even $50 \mathrm{~nm}$ could be done using times as short as $0.50 \mu \mathrm{s}$ and $0.55 \mu \mathrm{s}$. These good results are due to the use of the antistatic layer. ZEP520 resist has a sensitivity of $220 \mu \mathrm{C} / \mathrm{cm}^{2}$. According to this, the calculated dose for the field size $(600 \mu \mathrm{m} \times 600 \mu \mathrm{m})$, voltage and current of e-beam used as well as for the amount of dots per
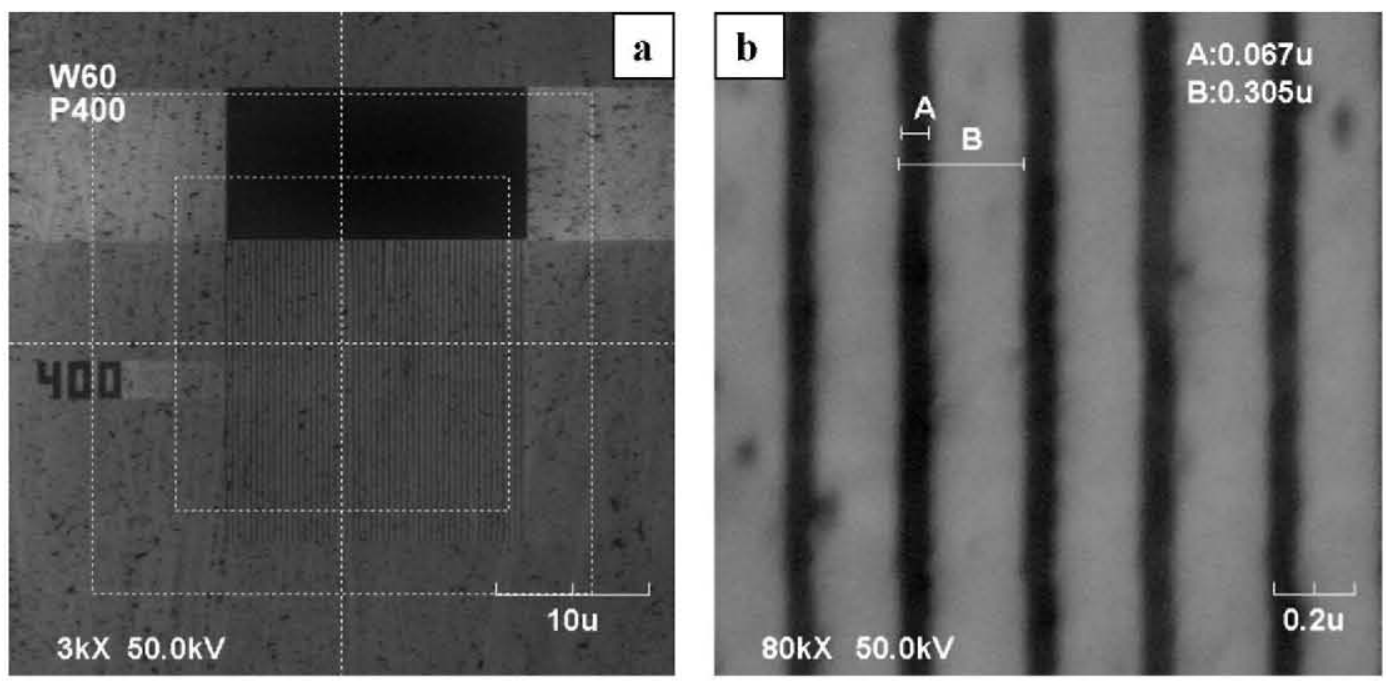

Fig. 10. Sapphire $\left(\mathrm{Al}_{2} \mathrm{O}_{3}\right.$ ) substrate: (a) $60 \mathrm{~nm}$ electrodes on a $400 \mathrm{~nm}$ pitch (dose time of $50 \mu \mathrm{s}$ ). (b) $60 \mathrm{~nm}$ electrodes on $300 \mathrm{~nm}$ pitch (dose time of $70 \mu \mathrm{s}$ ). 


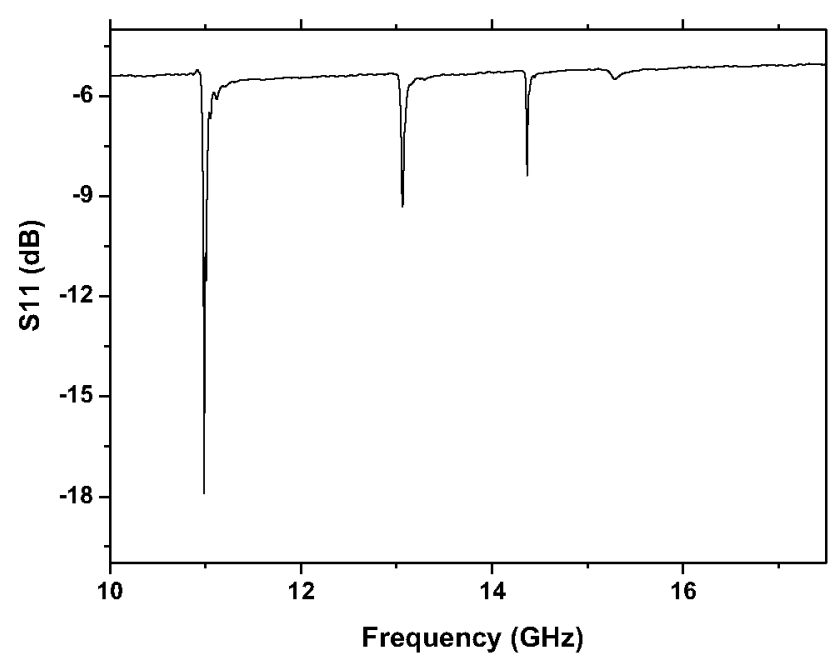

Fig. 11. Reflection coefficient of a $200 \mathrm{~nm}$ electrode width SAW resonator.

field used (60.000) should be $0.44 \mu \mathrm{s}$, close to the dose times used for patterning the IDTs. As displayed in Fig. 10, IDTs with $60 \mathrm{~nm}$ wide electrodes after lift-off can successfully be manufactured on the $\mathrm{AlN} / \mathrm{Al}_{2} \mathrm{O}_{3}$ system, by using the techniques described above.

\subsection{SAW device}

In order to exploit the procedures discussed above, SAW devices with electrodes widths from 100 to $200 \mathrm{~nm}$ were processed on an AlN/diamond heterostructure in order to increase the frequency at which they operate. During e-beam exposure, the AlN/diamond heterostructure is equal to the $\mathrm{Al}_{2} \mathrm{O}_{3}$ and $\mathrm{AlN} / \mathrm{Al}_{2} \mathrm{O}_{3}$ systems in terms of charge building effects. Thus the use of the antistatic layer was also required in this case.

AlN films $600 \mathrm{~nm}$ thick were deposited in a home built balanced magnetron sputter deposition system on microcrystalline diamond substrates. These substrates were fabricated by a chemical vapour deposition (CVD) and polished. The IDT of the device consists of 100 fingers with an electrode width of $200 \mathrm{~nm}$. Fig. 11 shows the reflection coefficient of a SAW resonator showing three resonances, the Rayleigh mode at $11.0 \mathrm{GHz}$, and some other confined modes at 13.1 and $14.4 \mathrm{GHz}$, respectively. Improved devices are under way to further increase the operating frequencies.

\section{Conclusions}

This work shows that line widths of $100 \mathrm{~nm}$ and above can easily be resolved using the calculated dose of resists. However, the manufacture of sub $100 \mathrm{~nm}$ lines requires higher doses depending on the substrate as well as on the finger width/pitch ratio. The dose time required for the fabrication of IDTs of variable line width and line pitch patterned by e-beam lithography has been determined for five substrates of different conductive character. Platinum, silicon and AlN/Si substrates do not require the use of an antistatic layer to obtain line widths below $100 \mathrm{~nm}$. However, e-beam patterning of these lines on insulating substrates $\left(\mathrm{AIN} / \mathrm{Al}_{2} \mathrm{O}_{3}\right.$ and $\left.\mathrm{Al}_{2} \mathrm{O}_{3}\right)$ requires the use of such layer. We have used this procedure to fabricate SAW devices on $\mathrm{AlN} /$ diamond structures, operating above $10 \mathrm{GHz}$.

\section{Acknowledgements}

GFI acknowledges the Spanish Ministerio de Ciencia e Innovación (MICINN) contract W050920B0. This work is supported by MICINN projects TEC2007-67065/MIC and TEC2010-19511.

\section{References}

Assouar, M.B., Elmazria, O., Kirsch, P., Mortet, V., Tiusan, C., Alnot, P., 2007. High-frequency surface acoustic wave devices based on AlN/diamond layered structure realized using e-beam lithography. Journal of Applied Physics 101, 114507.

Hatekeyama, H., Omori, T., Hashimoto, K., Yamaguchi, M., 2004. Fabrication of SAW devices using SEM-based electron beam lithography and lift-off technique for lab use. In: IEEE International Ultrasonics, Ferroelectrics, and Frequency Control Joint 50th Anniversary Conference, pp. 1896-1900.

Iriarte, G.F., 2009. Surface acoustic wave propagation characteristics of aluminium nitride thin films grown on polycrystalline diamond. Journal of Applied Physics 93 (12), 9604-9609.

Kirsh, P., Assouar, M.B., Mortet, V., Alnot, P. Elmazria, O, 2006.5 GHz surface acoustic wave devices based on aluminium nitride/diamond layered structure realized using electron beam lithography. Applied Physics Letters 88, 223504.

Müller, A., Neculoiu, D., Konstantinidis, G., Deligeorgis, G., Dinescu, A., Stavrinidis A., Cismaru, A., Dragoman, M., Stefanescu, A., 2010. SAW devices manufactured on GaN/Si for frequencies beyond $5 \mathrm{GHz}$. IEEE Electron Device Letters 31, $1398-1400$.

Neculoiu, D., Müller, A., Deligeorgis, G., Dinescu, A., Stavrinidis, A., Vasilache, D., Cismaru, A.M., Stan, G.E., Konstantinidis, G., 2009. AlN on silicon based surface acoustic wave resonators operating at $5 \mathrm{GHz}$. Electronics Letters $45,23$.

Palacios, T., Calle, F., Monroy, E., Grajal, J., Eickhoff, M., Ambacher, O., Prieto, C., 2002. Nanotechnology for SAW devices on AIN epilayers. Materials Science and Engineering B 93, 154-158.

Smith, H.I., 1974. Fabrication techniques for surface-acoustic-wave and thin-film optical devices. Proceeding of the IEEE 62 (10), 1361-1387.

Yamanouchi, K., Cho, Y., Meguro, T., 1988. SHF-range surface acoustic wave inter digital transducers using elect ron beam exposure. In: Proceeding IEEE Ultrasonic Symposium, pp. 115-118. 